\title{
ON THE BOUNDARY VALUES OF BLASCHKE PRODUCTS AND THEIR QUOTIENTS
}

\author{
CHUJI TANAKA
}

1. Introduction. Let $B(z)$ be the infinite Blaschke product:

$$
e^{i \lambda} z^{m} \prod_{n=1}^{+\infty} \bar{a}_{n}\left(a_{n}-z\right) /\left|a_{n}\right|\left(1-\bar{a}_{n} z\right),
$$

where $\lambda$ is a real constant, and $m$ is a non-negative integer, $0<\left|a_{n}\right|$ $<1, \sum_{n=1}^{+\infty}\left(1-\left|a_{n}\right|\right)<+\infty$. The object of this note is to establish the following two theorems.

THEOREM 1. (A) If the subsequence $\left\{a_{n_{k}}\right\}$ tends to $z=e^{i \phi}$ within the Stolz domain in such a manner that

$$
\lim _{k \rightarrow+\infty}\left|\left(a_{n_{k}}-a_{n_{k+1}}\right)\right| /\left|a_{n_{k}}-e^{i \phi}\right|=0,
$$

then the angular limit at $e^{i \phi}$ of $B(z)$ is 0 .

(B) If the subsequence $\left\{a_{n_{k}}\right\}$ tends to $z=e^{i \phi}$ within the circle: $\left|z-a e^{i \phi}\right| \leqq 1-a(0<a<1)$, in such a manner that

$$
\lim _{k \rightarrow+\infty} 1 / x_{k}^{2} \cdot\left|a_{n_{k}}-a_{n_{k+1}}\right|=0
$$

where $x_{k}=\min \left\{\left|a_{n_{k}}-e^{i \phi}\right|,\left|a_{n_{k+1}}-e^{i \phi}\right|\right\}$, then the angular limit at $e^{i \phi}$ of $B(z)$ is 0 .

As an application of Theorem 1 (A), we prove

THEOREM 2. There exists a meromorphic function $f(z)$ of bounded characteristic in $|z|<1$ represented by the quotient of two infinite Blaschke products such that

(1) $f(z)$ has infinite number of zeros and poles on $\arg (1-z)=-\vartheta$ and $\arg (1-z)=+\vartheta$ respectively $(0<\vartheta<\pi / 2)$.

$$
\lim _{z \rightarrow 1 ; \arg (1-z)=-\vartheta} f(z)=0, \quad \text { and } \lim _{z \rightarrow 1 ; \arg (1-z)=+\vartheta} f(z)=\infty .
$$

Remark. (1) O. Frostman [1, p. 109] was the first to construct an example of Blaschke product with the boundary value 0 , i.e., $B(z)=\prod_{n=1}^{+\infty}\left\{\left(1-1 / n^{2}\right)-z\right\} /\left\{1-\left(1-1 / n^{2}\right) z\right\}$, where $\lim _{r \rightarrow 1} B(r)=0$.

Received by the editors April 4, 1962. 
(2) By the well-known Iversen-Lindelöf theorem on asymptotic values, $f(z)$ of Theorem 2 has Picard's property in the sector $S:|\arg (1-z)| \leqq \vartheta<\pi / 2 ; w=f(z)$ takes every value $w$, except perhaps two, infinitely many times in $S$. On the other hand, $f\left(e^{i \theta}\right)$ is of modulus one almost everywhere on $|z|=1$.

(3) D. A. Storvick [3, p. 37] constructed a meromorphic function $f(z)$ defined by the quotient of two infinite Blaschke products such that $f(z) \rightarrow 0$ and $\infty$ as $z \rightarrow 1$ along the upper and lower oricycle: $r=\cos \theta$ respectively, $z=r e^{i \theta}$.

2. Proof of Theorem 1. (A) We decompose $B(z)$ as follows:

$$
B(z)=B_{1}(z) \cdot B_{2}(z),
$$

where $B_{1}(z)=\prod_{k=1}^{+\infty} \bar{a}_{n_{k}}\left(a_{n_{k}}-z\right) /\left|a_{n_{k}}\right|\left(1-\bar{a}_{n_{k}} z\right), \quad B_{2}(z)=B(z) / B_{1}(z)$. Since $|B(z)|<\left|B_{1}(z)\right|$ for $|z|<1$, it is sufficient to prove that the angular limit at $e^{i \phi}$ of $B_{1}(z)$ is 0 .

Without any loss of generality, we can assume that $\phi=0$. Put $z=1-r e^{i \theta}, a_{n_{k}}=b_{k}=1-r_{k} e^{i \theta_{k}}$. By a simple calculation,

$$
\begin{aligned}
& \left(b_{k}-z\right) /\left(1-\bar{b}_{k} z\right) \\
& =\left(b_{k}-z\right) / r_{k} e^{-i \theta_{k}} \cdot\left\{\left(e^{i 2 \theta_{k}}+1\right)-r e^{i \theta}+\left(b_{k}-z\right) / r_{k} e^{i \theta_{k}} \cdot e^{i 2 \theta_{k}}\right\}^{-1} .
\end{aligned}
$$

Let us denote by $l_{k}$ the segment connecting two points $b_{k}$ and $b_{k+1}$. If $z$ lies on $l_{k}$, we have evidently

$$
\left|b_{k}-z\right| \leqq\left|b_{k}-b_{k+1}\right|, \quad r \leqq \max \left(r_{k}, r_{k+1}\right) \text {. }
$$

By $\left|\theta_{k}\right| \leqq \vartheta<\pi / 2$, we get easily

$$
\left|e^{i 2 \theta_{k}}+1\right|>\sin (2 \vartheta) .^{1}
$$

By (2.1), (2.2) and (2.3)

$$
\begin{aligned}
\left|\left(b_{k}-z\right) /\left(1-b_{k} z\right)\right| & \leqq\left|\left(b_{k}-b_{k+1}\right)\right| /\left|1-b_{k}\right| \\
\cdot\{\sin (2 \vartheta) & \left.-\max \left(r_{k}, r_{k+1}\right)-\left|\left(b_{k}-b_{k+1}\right)\right| /\left|1-b_{k}\right|\right\}^{-1},
\end{aligned}
$$

so that, by the assumptions:

$$
\lim _{k \rightarrow \infty}\left|\left(b_{k}-b_{k+1}\right)\right| /\left|1-b_{k}\right|=0, \quad \lim _{k \rightarrow \infty} \max \left(r_{k}, r_{k+1}\right)=0,
$$

we obtain

$$
\lim _{z \rightarrow \infty}\left(b_{k}-z\right) /\left(1-b_{k} z\right)=0
$$

where $z \in l_{k}$. Since

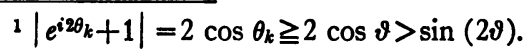




$$
\left|B_{1}(z)\right|<\left|\left(b_{k}-z\right) /\left(1-b_{k} z\right)\right| \quad \text { for any } k \text { and }|z|<1,
$$

by (2.4)

$$
\lim B_{1}(z)=0,
$$

as $z \rightarrow 1$ along $C=\bigcup_{k} l_{k}$. Hence, by Lindelöf's theorem [2, p. 5]

$$
\lim _{z \rightarrow 1} B_{1}(z)=0 \text {, }
$$

as $z \rightarrow 1$ inside a Stol $z$ domain with vertex at $z=1$, as was to be proved.

(B) Using the same notations as above, we get

(2.5) $\left(b_{k}-z\right) /\left(1-b_{k} z\right)=\left(b_{k}-z\right) / r r_{k} e^{i\left(\theta-\theta_{k}\right)} \cdot\left\{e^{i \theta_{k}} / r_{k}+e^{-i \theta} / r-1\right\}^{-1}$.

In the circle: $|z-a| \leqq 1-a\left(0<a<1, z=1-r e^{i \theta}\right)$, we have

$$
\frac{1}{2(1-a)} \leqq \cos \theta / r \text {. }
$$

If $z$ lies on $l_{k}$, by (2.5) and (2.6),

$$
\begin{aligned}
\left|\left(b_{k}-z\right) /\left(1-b_{k} z\right)\right| & \leqq\left|\left(b_{k}-z\right)\right| / r r_{k} \cdot\left\{\cos \theta_{k} / r_{k}+\cos \theta / r-1\right\}^{-1} \\
& \leqq(1 / a-1) \cdot y_{k} /\left(\min (r) \cdot x_{k}\right),
\end{aligned}
$$

where $y_{k}=\left|b_{k}-b_{k+1}\right|, x_{k}=\min \left(r_{k}, r_{k+1}\right), \min (r)=\min _{z \in l_{k}}|z-1|$. If $\min (r)=x_{k}$, we have

$$
\left|\left(b_{k}-z\right) /\left(1-\bar{b}_{k} z\right)\right| \leqq(1 / a-1) \cdot y_{k} / x_{k}^{2} .
$$

If $\min (r)<x_{k}$, we have easily

$$
\min (r) \geqq\left(x_{k}^{2}-\left(y_{k} / 2\right)^{2}\right)^{1 / 2},
$$

so that

$$
\left|\left(b_{k}-z\right) /\left(1-b_{k} z\right)\right| \leqq(1 / a-1) \cdot y_{k} / x_{k}^{2} \cdot\left\{1-\left(y_{k} / 2 x_{k}\right)^{2}\right\}^{-1 / 2} \text {. }
$$

In any case, by the assumption: $\lim _{k \rightarrow \infty} y_{k} / x_{\mathbf{k}}^{2}=0$, we have

$$
\lim _{z \rightarrow \infty}\left(b_{k}-z\right) /\left(1-b_{k} z\right)=0
$$

as $z$ on $l_{k}$. Hence, by entirely similar arguments as in (A),

$$
\lim _{z \rightarrow 1} B(z)=0
$$

as $z \rightarrow 1$ inside the Stolz domain with vertex at $z=1$.

3. Lemmas. To prove Theorem 2, we need two lemmas.

Lemma 1. Put 


$$
w(z)=(a-z)(1-a z) /(1-\bar{a} z)(\bar{a}-z),
$$

where $|a|<1, I(a)>0 .^{2}$ Then

$$
|w(z)|<1 \quad \text { for }|z|<1, I(z)>0 .
$$

Proof. $w(z)$ is regular in the upper semi-circle $D:|z| \leqq 1, I(z) \geqq 0$. On the boundary of $D$, we have evidently $|w|=1$. Hence, by the maximum-modulus principle, $|w(z)|<1$ for $|z|<1, I(z)>0$.

LEMma 2. In the domain $D:|z|<1, I(z) \geqq 0,|z-1| \leqq|a-1|$, where $|a|<1, I(a)>0$, we have

$$
|(1-a z) /(z-\bar{a})|<\exp \left(2 / \sin ^{2} \vartheta\right)
$$

where $\arg (1-a)=-\vartheta(0<\vartheta<\pi / 2)$.

Proof. By the inequality: $\log (1+x) \leqq x$ for $x \geqq 0$, for $|a|<1$, $|z| \leqq 1$ we obtain

$$
\begin{aligned}
\log |(1-a z) /(z-\bar{a})| & =\frac{1}{2} \log \left\{1+\left(1-|a|^{2}\right)\left(1-|z|^{2}\right) /|(z-\bar{a})|^{2}\right\} \\
& \leqq \frac{1}{2}\left(1-|a|^{2}\right)\left(1-|z|^{2}\right) /|(z-\bar{a})|^{2} \\
& <2|(1-a)(1-z)| /|(z-\bar{a})|^{2} .
\end{aligned}
$$

Hence

$\log |(1-a z) /(z-\bar{a})|<2|1-a|^{2} /|I(a)|^{2}=2 / \sin ^{2} \vartheta \quad$ for $z \in D$ because $|z-1| \leqq|a-1|,|z-\bar{a}| \geqq I(a)$ in $D$. Thus Lemma 2 is proved.

4. Proof of Theorem 2. Let the sequence $\left\{\epsilon_{n}\right\}$ be such that

$$
\begin{gathered}
\cos \vartheta>\epsilon_{1}>\epsilon_{2}>\cdots>\epsilon_{n}>\rightarrow 0, \\
\sum_{n=1}^{+\infty} \epsilon_{n}<+\infty \\
\lim _{n \rightarrow \infty} \epsilon_{n+1} / \epsilon_{n}=1 .
\end{gathered}
$$

Put $a_{n}=1-\epsilon_{n} \cdot e^{-i \vartheta}(0<\vartheta<\pi / 2)$. Then

$$
\left|a_{n}\right|<1, \quad I\left(a_{n}\right)>0 \quad \text { for } n \geqq 1 .
$$

The desired function $f(z)$ is given by $f(z)=B_{1}(z) / B_{2}(z)$, where

\footnotetext{
${ }^{2} I(a)$ is the imaginary part of $a$.
} 


$$
\begin{aligned}
& B_{1}(z)=\prod_{n=1}^{+\infty} \bar{a}_{n}\left(a_{n}-z\right) /\left|a_{n}\right|\left(1-\bar{a}_{n} z\right), \\
& B_{2}(z)=\prod_{n=1}^{+\infty} a_{n}\left(\bar{a}_{n}-z\right) /\left|a_{n}\right|\left(1-a_{n} z\right)
\end{aligned}
$$

Since $\sum_{n=1}^{+\infty} 1-\left|a_{n}\right|<\sum_{n=1}^{+\infty}\left|1-a_{n}\right|=\sum_{n=1}^{+\infty} \epsilon_{n}<+\infty$, the Blaschke products $B_{i}(z)(i=1,2)$ are convergent.

We can put

$$
f(z)=\prod_{n=1}^{+\infty} \bar{a}_{n} / a_{n} \cdot\left(a_{n}-z\right)\left(1-a_{n} z\right) /\left(1-\bar{a}_{n} z\right)\left(\bar{a}_{n}-z\right),
$$

so that, by Lemmas 1 and 2, we have

$$
|f(z)|<\left|\left(a_{k}-z\right) /\left(1-\bar{a}_{k} z\right)\right| \cdot \exp \left(2 / \sin ^{2} \vartheta\right)
$$

on the segment: $\arg (1-z)=-\vartheta,|1-z| \leqq \epsilon_{k}$. By (4.1)

$$
\left|\left(a_{k}-a_{k+1}\right) /\left(1-a_{k}\right)\right|=1-\epsilon_{k+1} / \epsilon_{k} \rightarrow 0 \quad \text { as } k \rightarrow+\infty .
$$

Hence, by (4.2) and arguments similar to those in the proof of Theorem $1(A)$,

$$
\lim _{z \rightarrow 1 ; \arg (1-z)=-\vartheta} f(z)=0 .
$$

Similarly

$$
\lim _{z \rightarrow 1 ; \arg (1-z)=+\vartheta} 1 / f(z)=0 .
$$

Thus Theorem 2 is completely established.

\section{REFERENCES}

1. O. Frostman, Potentiel d'équilibre et capacité des ensembles, Thèse, Lund, 1935.

2. K. Noshiro, Cluster sets, Springer, Berlin, 1960.

3. D. A. Storvick, On meromorphic functions of bounded characteristic, Proc. Amer. Math. Soc. 8 (1957), 32-38.

WASEDA UnIVERSITY, ToKYO 